\title{
Endständige Wasserfilter zur Prävention der Legionellose
}

\author{
Wirklich „Evidenz" oder alles nur „Eminenz"?
}

\section{Ines Kappstein}

Nosokomiale Legionellosen. In dieser Ausgabe von Krankenhaushygiene up2date finden Sie einen Artikel über die Bedeutung von Leitungswasser als Quelle für nosokomiale Infektionen (S. 261). Die bekannteste wasserassoziierte Infektion ist die Legionellose. Im Zusammenhang mit dieser Infektion ist es in Deutschland (aber nicht nur dort [1]) zu einer - von Zeit zu Zeit auch öffentlichen - Diskussion gekommen, deren hervorstechendes Merkmal die fehlende Rationalität ist.

Es wird einerseits so getan, als könne man Legionellen im Leitungsnetz von Krankenhäusern ganz einfach eliminieren, indem man nur die wassertechnischen Vorgaben der Deutschen Vereinigung des Gas- und Wasserfaches e.V. (DVGW, www.dvgw.de) umsetzt. Da aber andererseits auch zur Kenntnis genommen werden muss, dass dieses wünschenswerte Ziel offensichtlich doch nicht (immer) erreicht werden kann, wird der Einsatz endständiger Sterilwasserfilter für so genannte Risikobereiche als einzige Möglichkeit dargestellt, gefährdete Patienten vor dem Kontakt mit Legionellen zu schützen.

Wasserhygiene in Deutschland. Tonangebend auf dem Feld der Wasserhygiene ist in Deutschland die Trinkwasserkommission (TWK) des Bundesministeriums für Gesundheit (BMG) beim Umweltbundesamt (UBA). Im Bundesgesundheitsblatt führt ihr Vorsitzender als Beleg für „neue wissenschaftliche Erkenntnisse über die Bedeutung von Wasser als Infektionsquelle“ u. a. eine EU-Richtlinie und das Merkblatt W551 der DVGW, aber eben keine wissenschaftliche Literatur an [2]. Er weist darauf hin, dass nur mit Filtern legionellenfreies Wasser erreichbar sei, bleibt dafür aber den Beleg schuldig [3]. Diese Aussagen folgen nicht dem Prinzip der evidenzbasierten Medizin, wonach es nicht darum geht, dass ein Professor eine Auffassung vertritt, sondern dass wesentliche Aussagen mit Daten aus der wissenschaftlichen Literatur zu belegen sind [4].

Wissenschaftliche Evidenz? Die Befürworter eines Filtereinsatzes belegen die Effektivität endständiger Wasserfilter mit einer Übersichtsarbeit aus dem Hause des Herstellers der in Deutschland am weitesten verbreiteten Wasserfilter [5], ferner mit zwei (von diesem Hersteller gesponserten) mikrobiologischen Arbeiten [6,7]. Hierbei bleibt unerwähnt, dass in [7] ausdrücklich kontrollierte, langfristige Studien in verschiedenen Kliniken gefordert werden, um nach der in vitro-Wirksamkeit auch die klinische Effektivität der Filter zur Prävention nosokomialer Legionellen-Infektionen zu prüfen. Es werden gelegentlich nationale und internationale Leitlinien (von RKI, WHO, aus Frankreich) angeführt, in denen jedoch an keiner Stelle endständige Wasserfilter für die Prävention von Legionellosen auch nur erwähnt werden. Obwohl also wissenschaftliche Belege fehlen, wird zumindest implizit die bewiesene Wirksamkeit von Filtern zur Prävention von Legionellosen weiterhin behauptet [8].

Legionellosen trotz Filter. Mir sind Fälle von Legionellosen trotz Verwendung von Wasserfiltern bekannt (unpublizierte Daten). Darauf angesprochen, erläuterte die Firma (persönliche Mitteilung), dass dafür mit Sicherheit keine Insuffizienz der Filter, sondern z. B. eine retrograde Kontamination der Filterunterseite aus dem Siphon des Waschbeckens verantwortlich sein könne; eben damit sei auch die maximale Standzeit ihrer Filter begründet. Ferner könne die Filterunterseite durch das Reinigungspersonal kontaminiert werden. Außerdem, so wird weiterhin ausgeführt, gäbe es in jedem Bad Aerosolquellen, die mit endständigen Filtern nicht zu beeinflussen seien - wie etwa das WC bei der Betätigung der Spülung (!). In solchen Fällen von Legionellosen hätten Wasseruntersuchungen der Patientenumgebung durch firmeneigene Untersucher regelmäßig andere Wasserquellen (siehe oben) als Reservoir für den Legionellenkontakt der Erkrankten ergeben. Bei dieser Argumentation von Seiten des Herstellers drängt sich die Frage auf, warum man überhaupt Filter an den Wasserhähnen verwenden soll, wenn es doch anderweitig und unvermeidlich zu relevanten Legionellenkontakten kommen kann, denn die Filterunterseite kann immer durch hoch spritzendes Wasser oder die Hände von Personal oder Patien- 
ten kontaminiert werden, und auch Risikopatienten müssen nun einmal das WC benutzen.

Primärprävention. Im Zusammenhang mit nosokomialen Infektionen wird in Deutschland gerne auf die Bedeutung der Primärprävention verwiesen. Deren Effektivität muss allerdings auch nicht nur behauptet, sondern belegt sein. Wenn aber die Primärprävention von Legionellosen bedeuten soll, dass die Legionellen aus den Leitungsnetzen zu eliminieren sind, dann muss man einwenden, dass dies aller Erfahrung nach nicht möglich ist - auch nicht durch Umsetzung der empfohlenen technischen Maßnahmen. Das ist die ernüchternde Realität. Die Erfahrung zeigt, dass Legionellen in weit verzweigten Leitungsnetzen höchstens, wenn überhaupt, vermindert, aber nicht vollständig eliminiert werden können. Dies ist auch der Fall, wenn bei der Warmwasserbereitung und -verteilung alle technischen Empfehlungen umgesetzt werden: Das Wasser verlässt die Trinkwasserzentrale mit ca. $60^{\circ} \mathrm{C}$ und ist legionellenfrei und kommt aus der Zirkulation mit ca. $55^{\circ} \mathrm{C}$ wieder zurück und ist wieder legionellenfrei. Nicht frei von Legionellen ist dagegen oft das Wasser in den Wasserleitungen, die von der Ringleitung abzweigen, selbst wenn es noch mit ca. $55^{\circ} \mathrm{C}$ an den Wasserhähnen ankommt. „Primärprävention“ erschöpft sich dann lediglich 1 . in der wiederholten Beprobung von Leitungswasser sowie ggf. 2. in der Installation zentraler Anlagen, die an den Zapfstellen keine Wirkung zeigen, weil sie nicht in ausreichendem Maße in der Lage sind, Legionellen in den Biofilmen peripherer Leitungen zu inaktivieren, sowie 3. in der langfristig äußerst kostenintensiven Installation endständiger Wasserfilter, die doch nur ein falsches Gefühl von Sicherheit vermitteln.

Rationale Prävention von Legionellosen. Für die Prävention von Legionellosen sind aber (außer in Situationen mit nosokomialen Legionellosefällen) weder häufige Wasseruntersuchungen erforderlich [9], die immer nur eine „Momentaufnahme“ wiedergeben, noch Grenzwerte für Legionellen, die willkürlich - und nicht auf der Basis epidemiologischer Studien - festgesetzt wurden. Dies trifft sowohl auf die Grenzwerte der DVGW als auch der TKW zu [10]. Wichtig ist vielmehr das Wissen, dass Legionellen nun einmal im Wasser vorkommen (können), weshalb man 1. Risikopatienten durch Nutzungseinschränkung vor einem Kontakt mit Leitungswasser im Bereich der oberen Atemwege schützen muss und 2. insbesondere bei diesen Patienten bei Auftreten einer Pneumonie Legionellen immer sofort in das diagnostische und therapeutische Spektrum einbeziehen muss $[1,3,9]$.

Offene Diskussion. Eine Industrie-unabhängige Diskussion über Wasserfilter ist notwendig, weil sie den wissenschaftlichen Diskurs fördert - noch dazu über ein in Deutschland geradezu tabuisiertes Thema (denn viele Kliniken haben sogar Angst, über den Nachweis von Legionellen im Wasser zu sprechen, von Infektionsfällen gar nicht zu reden). Durch eine solche Diskussion würde zudem verhindert, dass einzelne Themen nur von Interessengruppen besetzt werden, zu denen im Übrigen nicht nur die einschlägige Industrie, sondern auch mikrobiologische und HygieneLabore gehören. Diese fordern in den von ihnen beratenen Kliniken die (in dieser Weise durch die Trinkwasserverordnung nicht gedeckte) Beprobung sämtlicher Wasserleitungen (inkl. Feuerlöschleitungen), um Legionellen zu suchen und um bei Legionellennachweis, der regelmäßig erfolgt, weitere Probenserien zu untersuchen. Diese Laboruntersuchungen kosten die Kliniken sehr viel Geld, während der primärpräventive Effekt dieser Maßnahme unbelegt ist [9]. Umso irritierender ist es, dass die TWK erst kürzlich Empfehlungen für Probennahmen publiziert hat, deren Umfang in keiner Weise durch entsprechende Daten gestützt, also nicht „evidence-based“ ist und die damit nicht den „Stand der medizinischen Wissenschaft" wiedergeben [11]. Ganz abgesehen davon legt die TWK immer noch das Hauptgewicht auf Duschen und hat somit offenbar nicht zur Kenntnis genommen, dass es keine Belege dafür gibt, dass Duschen mit einem höheren Legionelloserisiko verbunden ist als der Kontakt mit Leitungswasser an einem Wasserhahn [10].

„Evidenz“ oder „Eminenz“? Die Titel-Frage kann also dahingehend beantwortet werden, dass es bislang keine wissenschaftlichen Belege (= Evidenz) für die klinische (und nur diese ist entscheidend) Wirksamkeit endständiger Wasserfilter gibt, sondern lediglich Empfehlungen einzelner „Fachleute“, meist Hygieniker (= „Eminenz“). Somit handelt es sich um den niedrigsten Evidenzgrad, auch gobsat genannt: „Good old boys sat arround a table“. Aus der Entscheidung, endständige Wasserfilter aufgrund dieser Datenlage nicht einzusetzen, lassen sich ebenso wenig „haftungsrechtliche Konsequenzen“ [11] ableiten wie eine „ethisch nicht vertretbare Haltung“ begründen. 


\section{Literatur}

1 Sabria M, Yu VL. Hospital-acquired legionellosis: solutions for a preventable infection. Lancet Infect Dis 2002; 2: $368-373$

2 Exner M, Kistemann T. Bedeutung der Verordnung über die Qualität von Wasser für den menschlichen Gebrauch (Trinkwasserverordnung 2001) für die Krankenhaushygiene. Bundesgesundheitsbl - Gesundheitsforsch - Gesundheitsschutz 2004; 47 : $384-391$

3 Kappstein I. Wasserfilter zur Prävention nosokomialer Legionellosen? Dtsch Med Wochenschr 2006; 131: 2789-2792

4 Greenhalgh T. Einführung in die Evidence-based Medicine. Kritische Beurteilung klinischer Studien als Basis einer rationalen Medizin. Bern: Verlag Hans Huber, 2000

5 Ortolano GA, McAlister MB, Angelbeck JA et al. Hospital water point-of-use filtration: a complimentary strategy to reduce the risk of nosocomial infection. Am J Infect Control 2005; 33: S1-9

6 Vonberg RP, Eckmanns T, Bruderek J et al. Use of terminal tap water filter systems for prevention of nosocomial legionellosis. J Hosp Infect 2005; 60: $159-162$

7 Sheffer PJ, Stout JE, Wagener MM et al. Efficacy of new point-of-use water filter for preventing exposure to Legionella and waterborne bacteria. Am J Infect Control 2005; 33: S20- 25

8 Exner M, Kramer A, Kistemann T et al. Wasser als Infektionsquelle in medizinischen Einrichtungen, Prävention und Kontrolle. Bundesgesundheitsbl Gesundheitsforsch - Gesundheitsschutz 2007; 50: $302-311$

9 Centers for Disease Control and Prevention. Guidelines for environmental infection control in healthcare facilities: recommendations of CDC and the Healthcare Infection Control Practices Advisory Committee (HICPAC). MMWR 2003; 52: 1-48

10 Umweltbundesamt. Empfehlung des Umweltbundesamtes nach Anhörung der Trinkwasserkommission des Bundesministeriums für Gesundheit. Bundesgesundheitsbl - Gesundheitsforsch Gesundheitsschutz 2006; 49: 697-700

11 Ulsenheimer K. Haftungsrechtliche Bedeutung von Leitlinien. Krankenh.hyg. up2date 2006; 1: 169 175 